\title{
THE RESULTS OF EARLY VERSUS LATE SURGERY IN TRAUMATIC CERVICAL FACET JOINT DISLOCATION: A RETROSPECTIVE STUDY
}

\author{
(1) Mustafa Sakar, (1) Toghrul Javadov, (1) Can Kıvrak, (1) Yahya Güvenç, (1) Ferhat Harman
}

Marmara University Faculty of Medicine, Department of Neurosurgery, İstanbul, Turkey

\begin{abstract}
Objective: Various strategies are suggested for facet joint dislocations after cervical trauma. The effect of timing of surgery on neurological outcome is controversial, both early and late surgeries have advantages and disadvantages. We aimed to investigate the neurological results of early versus late surgery for traumatic subaxial cervical facet joint dislocations. We also aimed to investigate the effect of unilateral and bilateral injuries on neurological recovery.

Materials and Methods: The data of 23 consecutive patients with facet joint dislocation between 2013-2020 were retrospectively analyzed. The data of age, gender, spinal level and side, surgical strategy, surgical timing, neurological status, and prognosis were collected from medical reports. Surgery within the first 24 hours of trauma was defined as early surgery and surgery after 24 hours of trauma was defined as late surgery. The effect of timing of surgery and unilateral or bilateral nature of the injury on neurological outcome were investigated at one-year follow-up. Results: In a total of 19 patients with neurological deficits, early surgery resulted in neurological recovery in 7 of 12 patients ( $p=0.001)$, and late surgery resulted in neurological recovery in 2 of 7 patients $(p=0.135)$. While 8 of 12 patients with unilateral dislocation showed neurological recovery $(p=0.002)$, only 3 of 11 patients with bilateral facet joint dislocations showed neurological recovery $(p=0.061)$.

Conclusion: Early surgery may result in better neurological outcomes at one-year follow up than late surgery. Patients with unilateral facet joint dislocation have better prognosis than bilateral injuries in terms of neurological recovery.
\end{abstract}

Keywords: Cervical trauma, subaxial cervical spine, facet joint dislocation, early surgery, traumatic disc herniation

\section{INTRODUCTION}

Trauma to the cervical spine is commonly related to motor vehicle accidents in young patients and falls in the elderly ${ }^{(1)}$. Nearly half of the cervical spinal injuries take place at $\mathrm{C} 5-\mathrm{C} 7$ levels and these segments are the most commonly affected levels ${ }^{(2)}$. Cervical trauma can result in fractures, traumatic disc herniations, listhesis, facet joint dislocations, and ligamentous injuries. They may lead to cervical instability and neurological deficits which may lead to serious morbidities and even mortality. Various treatment options are suggested for facet joint dislocation in literature including closed and open reductions. While the closed reduction is non-invasive and formerly accepted as advantageous, it is believed that it may lead to worsening of neurological deficits and open surgical treatments are more commonly adopted recently ${ }^{(3)}$. The most obvious indications for surgery are cervical instability and neurological deficits ${ }^{(4)}$. Surgical treatment for subaxial cervical trauma aims to decompress the spinal cord and roots, and also restore adequate cervical alignment and stability ${ }^{(5)}$. When surgical treatment is the choice, the timing of the surgery is another controversy. Whether early surgery is better for neurological deficits is an ongoing debate. In this study, we aim to investigate the neurological results of early versus late surgery for traumatic subaxial cervical facet joint dislocations in both unilateral and bilateral cases. We also aim to investigate the effect of unilateral and bilateral injuries on neurological recovery.

\section{MATERIALS AND METHODS}

This retrospective study was performed in accordance with the "Declaration of Helsinki". This study is approved by the Local Ethics Committee of Marmara University, by decision number 09.2021.667. This study included surgically treated patients admitted to our tertiary center between 2013 and 2020, with facet joint dislocation after trauma in the subaxial cervical spine. 
Age, gender, spinal level, and side, surgical strategy, surgical timing, neurological status, and prognosis were collected retrospectively. American Spinal Cord Injury Association (ASIA) scoring ${ }^{(6)}$ was used for neurological assessment at preoperatively and postoperatively at 12 months. A positive change of one level in ASIA scoring was accepted as neurological recovery. All patients were operated on using anterior, posterior, or a combination of anterior and posterior techniques aiming to achieve appropriate spinal cord decompression and alignment of the cervical spine. Early surgery was defined as the surgical procedure accomplished within the first 24 hours of trauma and late surgery was defined as the surgeries performed after 24 hours of trauma. The effect of the timing of surgery on the neurological outcome is investigated along with the unilaterality or bilaterality of the injury.

\section{Statistical Analysis}

Statistical analysis was accomplished using SPSS statistics software version SPSS 25.0 (SPSS Inc., IBM, NY,USA). ASIA scoring and uni-or-bilaterality were used as dependent variables in separate analyses, and Friedman Test was employed. P-values lower than 0.05 were considered statistically significant.

\section{RESULTS}

Twenty-three patients were included in the study. All had traumatic subaxial cervical facet joint dislocations and etiology included motor vehicle accidents, falls, and external mass hits. After careful examination, all patients were radiologically assessed using computed tomography (CT) and magnetic resonance imaging (MRI) including short tau inversion recovery. There were 16 males and 7 females, and their ages ranged between 17 and 77 (mean 49.17). Injured segments were C3-4 in one, C4-5 in 3, C5-6 in 9, and C6-7 in 10 patients. Twelve patients had unilateral and 11 patients had bilateral facet joint dislocations. There was traumatic disc herniation in 8 patients and this finding guided surgical strategy in most cases. Six of these 8 patients were operated on using the anterior approach only, and the remaining 2 patients were operated on with combined anterior and posterior approaches. One patient had an anterior approach but did not have a traumatic disc herniation. The remaining 14 patients without traumatic disc herniation were operated on using the posterior approach. One patient having ankylosing spondylitis as comorbidity, who was operated on using the anterior approach, required revision surgery due to instability one month after initial surgery, and posterior fixation was performed. Early physical therapy and rehabilitation were initiated in all patients on postoperative day 1.

ASIA scoring preoperatively was $E$ in 4 patients, $D$ for 5 patients, C for 5 patients, and B for 9 patients. In 4 ASIA E patients, scoring was $E$ for all at one year follow-up. In 5 ASIA D patients, 4 had ASIA E and 1 remained ASIA D in one year follow-up. In 5 ASIA $C$ patients, 3 had ASIA D and 2 remained ASIA C in one year follow-up. In 9 patients with ASIA B, 1 patient had ASIA E, 1 patient had ASIA $C$ and 7 remained ASIA B at one year followup (Table 1).

The early surgery group included 14 patients and the late surgery group included 9 patients. Twelve of 14 patients had neurological deficits in the early surgery group and seven of these 12 patients (30\% of total) showed neurological recovery. In the late surgery group, 7 of 9 patients had a neurological deficit and only 2 of them ( $8.6 \%$ of total) showed neurological recovery.

In total, 19 patients had neurological deficits preoperatively. The early surgery group included 12 patients and the late surgery group included 7 patients, respectively. While 7 of 12 patients (58\%) in the early surgery group showed neurological recovery, only 2 of 7 patients (28\%) in the late surgery group showed neurological recovery. In the early surgery group, postoperative one-year ASIA scores were significantly better than preoperative ASIA scores $(p=0.001)$, however, in the late surgery group, there were no significant differences between pre-and postoperative ASIA scores ( $p=0.135$ ) (Table 2).

While 8 of 12 patients with unilateral facet joint dislocation had neurological deficits (66\%), 11 out of 11 patients with bilateral facet joint dislocation had neurological deficits (100\%). In patients with unilateral facet joint dislocation with neurological deficit, 6 of 8 (75\%) showed neurological recovery. Their postoperative one-year ASIA scores were significantly better than preoperative ASIA scores $(p=0.002)$. In patients with bilateral facet joint dislocation with neurological deficit, 3 of 11 patients (27\%) showed neurological recovery. There were no significant differences between preoperative and postoperative ASIA scores in this group ( $p=0.061)$ (Table 3).

\section{DISCUSSION}

Instability and neurological deficits are the main indications of surgery after traumatic facet joint dislocations in the cervical spine. While a thorough neurological examination is of paramount importance in cervical spinal trauma, imaging modalities including $C T$ and MRI may delineate the injuries like facet joint alignment, traumatic disc herniation, posterior ligamentous disruptures, and bony fractures ${ }^{(7-9)}$. Traumatic disc herniation accompanying facet joint dislocation plays a very important role in decision making for the type of surgery, because a posterior approach in the presence of anterior disc herniation is reported to have a high risk for secondary/ iatrogenic spinal cord injuries ${ }^{(2,4,10)}$. The present study included 19 patients with neurological deficits and 8 of them showed evidence of traumatic disc herniation on MRI. Two of them had combined anterior and posterior approaches and 6 had anterioronly approach. Only one patient without disc herniation was operated on using the anterior approach and the remaining 14 patients had a posterior approach. This finding also shows the importance of the presence of traumatic disc herniation in decision-making for the approach to use. 
Table 1. Descriptive data for 23 patients

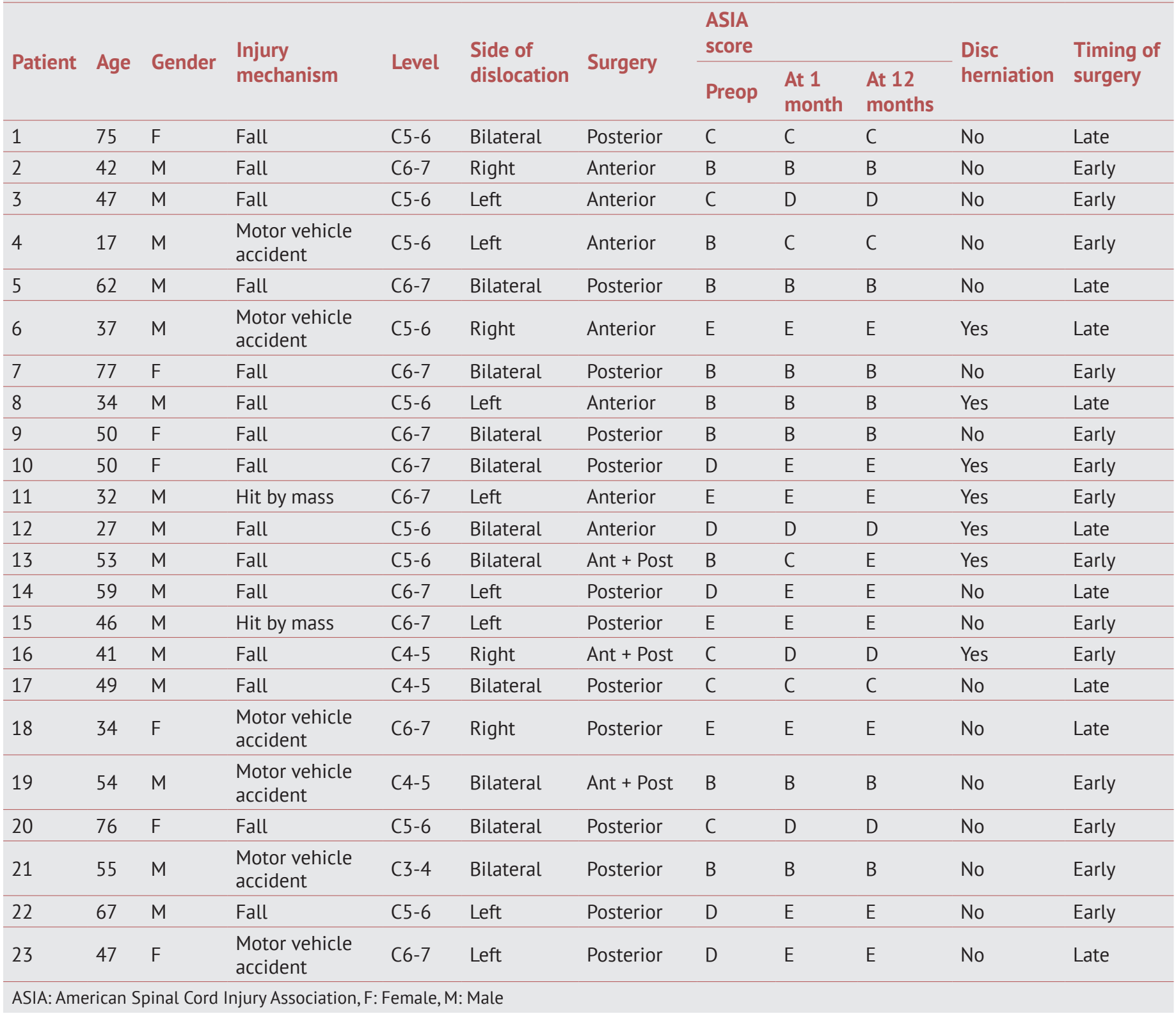

Table 2. The outcomes for patients having neurological deficit at one year follow-up with early and late surgeries

\begin{tabular}{llll}
\hline Timing of surgery & Total & Neurological recovery & p-value \\
\hline Early surgery & 12 & 7 & 0.001 \\
\hline Late surgery & 7 & 2 & 0.135
\end{tabular}

Table 3. The outcome for patients having neurological deficit at one year follow-up with unilateral and bilateral facet joint dislocations

\begin{tabular}{llll}
\hline Side of dislocation & Total & Neurological recovery & p-value \\
\hline Unilateral & 8 & 6 & 0.002 \\
\hline Bilateral & 11 & 3 & 0.061 \\
\hline
\end{tabular}


Closed or open reduction for cervical facet joint dislocation is still a controversial topic. Closed reduction is reported to have a success rate as high as $98 \%{ }^{(11)}$, although it is reported with much lower success rates in bilateral cases ${ }^{(12)}$. It may lead to worsening neurological function in patients who also have traumatic disc herniation ${ }^{(12,13)}$. Although the closed reduction is regarded as a non-invasive measure, open surgeries have the advantages of being safe, reliable, easy to perform, and lesser secondary/iatrogenic injuries ${ }^{(14-20)}$. Closed reduction is suggested in one study for conscious, cooperated, neurologically intact patients without traumatic disc herniation and posterior ligamentous injury ${ }^{(9)}$.

The posterior approach utilizes decompression from the posterior by removing bony fragments and disrupted ligaments, and has the advantage of direct reduction of the facet joints ${ }^{(10,21)}$. It may serve as a solid stabilization and fusion method. It also has disadvantages including more dissection of soft tissues, more segments needed for fusion, and a higher risk of complication in the presence of traumatic disc herniation or any other ventral compressions ${ }^{(22)}$. The anterior approach provides better cervical lordosis, fewer problems associated with scar tissue healing and postoperative pain ${ }^{(10)}$. It also allows decompression of traumatic disc herniation or any other ventral compressions ${ }^{(23)}$. On the other hand, the anterior approach may fail to re-align the facet joints properly and this distraction maneuver may also lead to secondary/ iatrogenic injury to the spinal cord ${ }^{(24)}$. Combined anterior and posterior approaches best reduce the risk of secondary/ iatrogenic injuries and are used in complex situations ${ }^{(10)}$. We did not use closed reduction in any of the patients in this study. In the case of ventral compression caused by a traumatic disc herniation or other bony fragments, an anterior approach was chosen. After successful anterior decompression, the reduction was also attempted anteriorly. In 6 patients, the anterior approach resulted in successful decompression and reduction, and surgery was completed with anterior fusion (Figure 1). However, in 3 patients, an anterior reduction attempt was failed and a posterior approach was added for reduction and appropriate cervical alignment (Figure 2). In the remaining 14 patients, there was no anterior compression and a posterioronly approach was used successfully for reduction (Figure 3). Patients with bilateral facet joint dislocation have more serious neurological deficits than patients with unilateral facet joint dislocations ${ }^{(25,26)}$. During the trauma, higher forces and excessive hyperflexion are the causes for bilateral facet dislocation, and this is also suggested as the cause for more serious neurological injuries in bilateral facet joint dislocations than unilateral facet joints dislocations ${ }^{(25,26)}$. While there were 11 patients in our study with bilateral facet joint dislocation and all had neurological deficits, there were 12 patients with unilateral facet joint dislocation and 8 of them had neurological deficits. This is concordant with recent literature.

Early or late surgery is also another controversial issue. Some reports suggest no difference in neurological function between
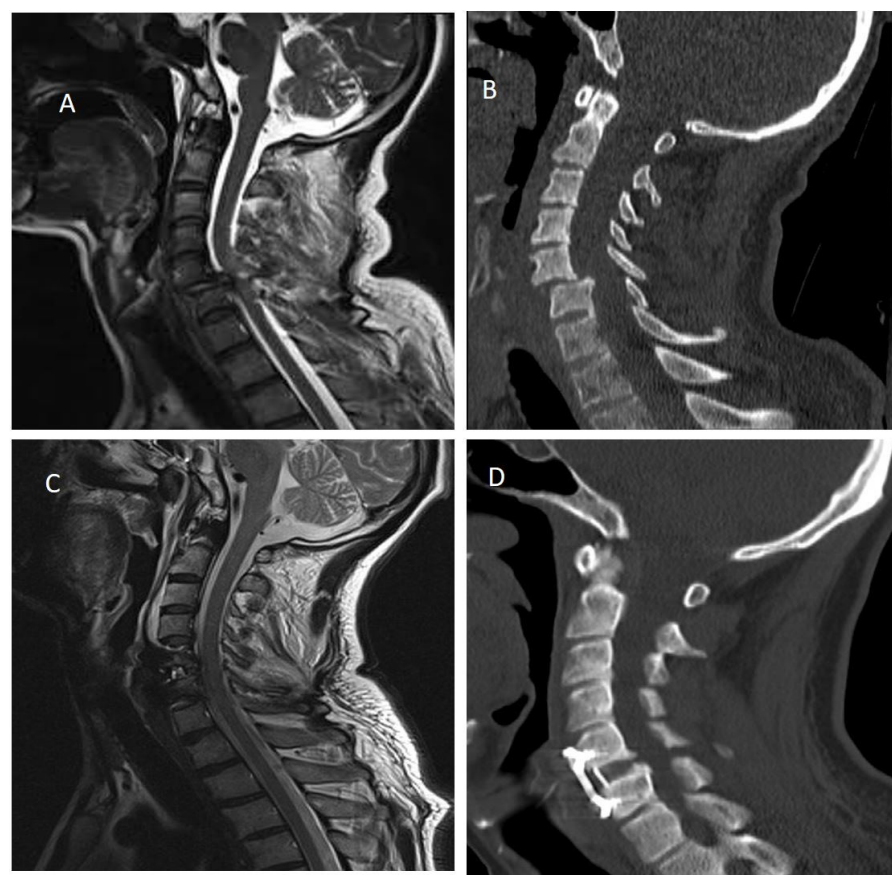

Figure 1. Radiological imaging of a patient operated anteriorly. A) Preoperative T2-weighted sagittal MRI showing anterolisthesis at C5-6 level. Traumatic disc herniation is evident at (5-6. B) Preoperative CT showing anterolisthesis at (5-6. C) Postoperative T2-weighted MRI imaging artifact at operated level and proper decompression of spinal cord. Hyperintensity at the level indicates spinal cord injury at C5-6 levels. D) Postoperative CT shows proper alignment of cervical spine and fusion material at the operated level

MRI: Magnetic resonance imaging, CT: Computed tomography

early and late surgeries ${ }^{(27)}$. It is also reported that in patients with complete neurological functional loss, early surgery just affects the hospital stay and has no effect on neurological function $^{(28)}$. On the other hand, several reports are suggesting early decompression and stabilization are associated with favorable outcomes for neurological function ${ }^{(3,29-34)}$. In Surgical Timing in Acute Spinal Cord Injury Study, it is shown that the positive effects of early surgery within the first 24 hours of cervical trauma on neurological recovery are more commonly observed than late surgery ${ }^{(35)}$. In a multi-center study, early surgery is found to have a direct association with a good prognosis in patients with complete motor paralysis ${ }^{(36)}$. In our study, results of early surgery favor a better neurological outcome in patients with traumatic facet joint dislocations with neurological deficits. While early surgery resulted in better neurological outcomes in 7 of 12 patients (58\%) at one-year follow-up, late surgery resulted in better neurological outcomes in only 2 of 7 patients (28\%) at one-year follow-up.

\section{Study Limitations}

Retrospective and non-randomized nature is the main limitation of the study. The relatively small cohort is also another limitation. Although it is difficult, prospective randomized 

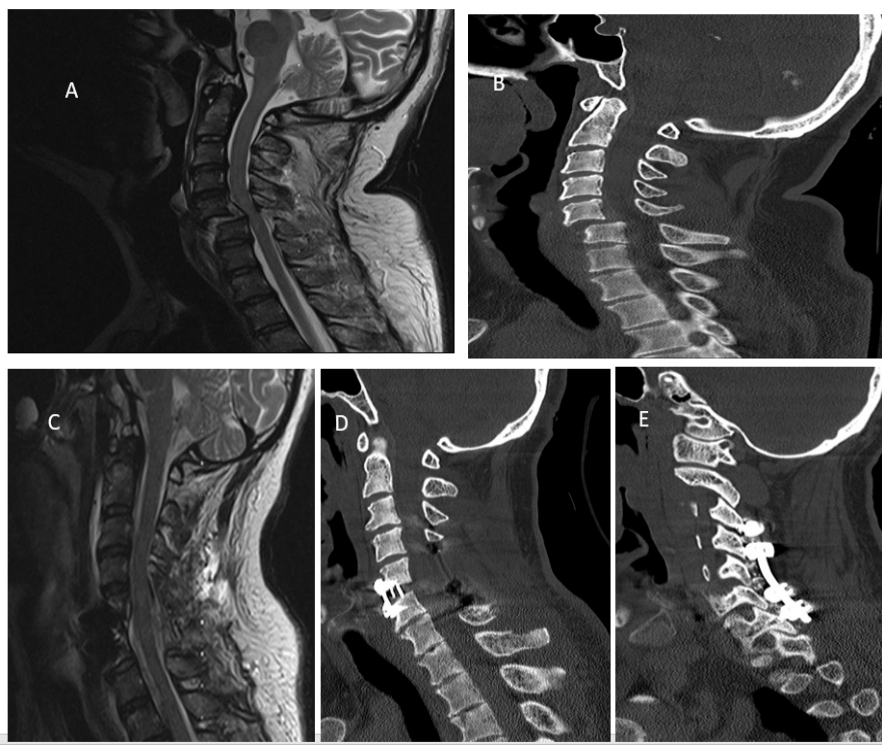

Figure 2. Radiological imaging of a patient operated both anteriorly and posteriorly. A) Preoperative T2-weighted sagittal MRI showing anterolisthesis at C5-6 level. B) Preoperative CT showing anterolisthesis at (5-6. C) Postoperative midsagittal T2-weighted MRI section shows proper decompression of apinal cord. Hyperintensity at the level indicates spinal cord injury at C5-6 levels. D) Postoperative CT shows proper alignment of cervical spine and anterior fusion material at the operated level. E) Post-operative CT shows proper alignment of cervical spine and posterior fusion material at the operated level

MRI: Magnetic resonance imaging, CT: Computed tomography
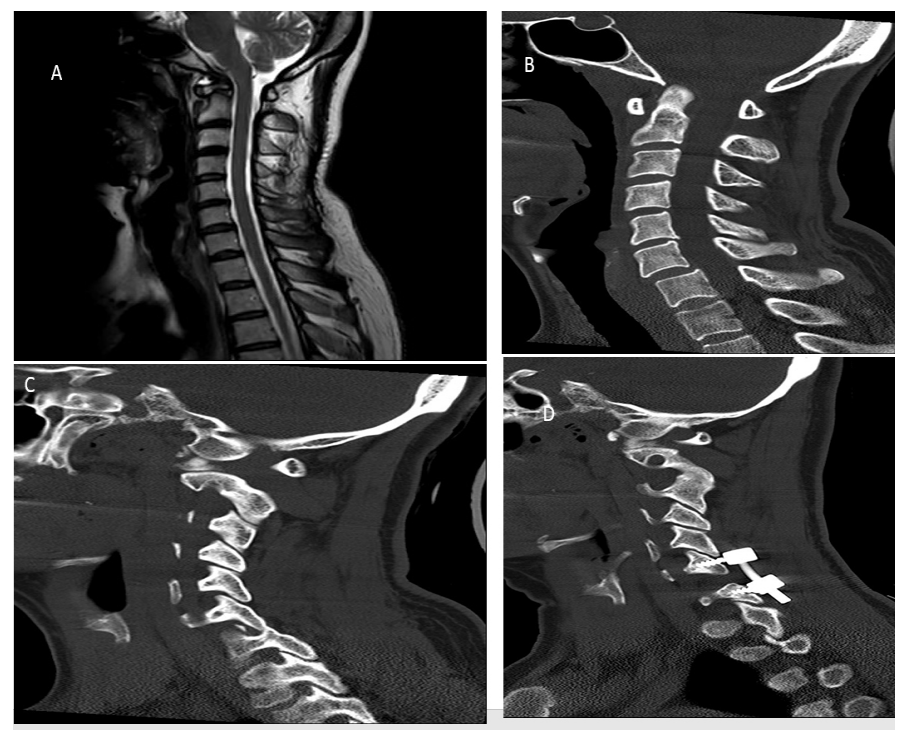

Figure 3. Radiological imaging of a patient operated posteriorly with unilateral facet joint dislocation. A) Preoperative midsagittal T2-weighted MRI section shows no abnormalities. B) Preoperative midsagittal CT section shows slight anterolisthesis. C) Preoperative sagittal CT section shows dislocated facet joint at C6-7 levels. D) Postoperative $\mathrm{CT}$ shows reduction of facet joints and posterior fusion material at the operated level

MRI: Magnetic resonance imaging, CT: Computed tomography studies with larger patient numbers may thoroughly investigate the effects of early surgery in facet joint dislocation regarding the neurological outcome.

\section{CONCLUSION}

In patients with facet joint dislocation, early surgery within the first 24 hours of cervical trauma may result in better neurological outcomes in the one-year follow-up than late surgery. Patients with unilateral facet joint dislocation have a better prognosis than patients with bilateral facet joint dislocation after cervical trauma in terms of neurological recovery.

\section{Ethics}

Ethics Committee Approval: This study is approved by the Local Ethics Committee of Marmara University, by decision number 09.2021.667.

Informed Consent: Retrospective study.

Peer-review: Internally peer-reviewed.

\section{Authorship Contributions}

Concept: M.S., Y.G., F.H., Design: M.S., Y.G., F.H., Data Collection or Processing: T.J., C.K., Analysis or Interpretation: M.S., T.J., C.K., Literature Search: T.J., C.K., Writing: M.S., T.J., C.K.

Conflict of Interest: The authors declare that they have no conflict of interest.

Financial Disclosure: The authors declared that this study received no financial support.

\section{REFERENCES}

1. Rafet Ö, Hanalioğlu Ş, Güneş SÖ, Yaman B, Türkoğlu ME, Ergün BR. Traumatic fractures and dislocations of subaxial cervical spine: one center results. Cukurova Med J. 2017;43:122-30.

2. Joaquim A, Lawrence B, Daubs M, Brodke D, Patel A. Evaluation of the subaxial injury classification system. J Craniovertebr Junction Spine. 2011;2:67-72.

3. Ordonez B], Benzel EC, Naderi S, Weller SJ. Cervical facet dislocation: techniques for ventral reduction and stabilization. I Neurosurg. 2000;92:18-23.

4. Aydın HE, Özbek Z, Vural M, Arslantaş A. Surgical management of subaxial cervical spine trauma: a case report. Meandros Med Dent J. 2015;16:25-9.

5. Joaquim AF, Patel AA. Subaxial cervical spine trauma: evaluation and surgical decision-making. Global Spine J. 2014;4:63-9.

6. Roberts TT, Leonard GR, Cepela DJ. Classifications in brief: American Spinal Injury Association (ASIA) Impairment Scale. Clin Orthop Relat Res. 2017;475:1499-504.

7. Du W, Wang C, Tan J, Shen B, Ni S, Zheng Y. Management of subaxial cervical facet dislocation through anterior approach monitored by spinal cord evoked potential. Spine (Phila Pa 1976). 2014;39:48-52.

8. Benzel EC, Hart BL, Ball PA, Baldwin NG, Orrison WW, Espinosa MC. Magnetic resonance imaging for the evaluation of patients with occult cervical spine injury. J Neurosurg. 1996;85:824-9.

9. Kwon BK, Vaccaro AR, Grauer JN, Fisher CG, Dvorak MF. Subaxial cervical spine trauma. J Am Acad Orthop Surg. 2006;14:78-89.

10. Lins CC, Prado DT, Joaquim AF. Surgical treatment of traumatic cervical facet dislocation: anterior, posterior or combined approaches? Arq Neuropsiquiatr. 2016;74:745-9.

11. Star AM, Jones AA, Cotler JM, Balderston RA, Sinha R. Immediate closed reduction of cervical spine dislocations using traction. Spine (Phila Pa 1976). 1990;15:1068-72. 
12. Zaveri G, Das G. Management of sub-axial cervical spine injuries. Indian J Orthop. 2017;51:633-52.

13. Eismont FJ, Arena MJ, Green BA. Extrusion of an intervertebral disc associated with traumatic subluxation or dislocation of cervical facets. Case report. J Bone Joint Surg Am. 1991;73:1555-60.

14. Zhou Y, Zhou Z, Liu L, Cao X. Management of irreducible unilateral facet joint dislocations in subaxial cervical spine: two case reports and a review of the literature. J Med Case Rep. 2018;12:74.

15. Alexander $\mathrm{E}$, Davis $\mathrm{CH}$, Forsyth HF. Reduction and fusion of fracture dislocation of the cervical spine. J Neurosurg. 1967;27:588-91.

16. Beyer C, Cabanela M, Berquist T. Unilateral facet dislocations and fracture-dislocations of the cervical spine. J Bone Joint Surg Br. 1991;73:977-81.

17. Caspar W, Barbier DD, Klara PM. Anterior cervical fusion and Caspar plate stabilization for cervical trauma. Neurosurgery. 1989;25:491-502.

18. Hadley MN, Fitzpatrick BC, Sonntag VK, Browner CM. Facet fracture-dislocation injuries of the cervical spine. Neurosurgery. 1992;30:661-6.

19. Rorabeck C, Rock M, Hawkins R, Bourne R. Unilateral facet dislocation of the cervical spine. An analysis of the results of treatment in 26 patients. Spine (Phila Pa 1976). 1987;12:23-7.

20. Shapiro SA. Management of unilateral locked facet of the cervical spine. Neurosurgery. 1993;33:832-7.

21. Kandziora F, Pflugmacher R, Scholz M, Schnake K, Putzier M, Khodadadyan-Klostermann $C$, et al. Posterior stabilization of subaxial cervical spine trauma: indications and techniques. Injury. 2005;36(Suppl 2):B36-43. doi: 10.1016/j.injury.2005.06.013.

22. Ye ZW, Yang SH, Chen BJ, Xiong LM, Xu JZ, He QY. Treatment of traumatic spondylolisthesis of the lower cervical spine with concomitant bilateral facet dislocations: risk of respiratory deterioration. Clin Neurol Neurosurg. 2014;123:96-101.

23. Berry C, Rao R. Compressive flexion and vertical compression injuries of the subaxial cervical spine. Semin Spine Surg. 2013;25:36-44.

24. Paxinos O, Ghanayem AJ, Zindrick MR, Voronov LI, Havey RM, Carandang $G$, et al. Anterior cervical discectomy and fusion with a locked plate and wedged graft effectively stabilizes flexiondistraction stage-3 injury in the lower cervical spine: a biomechanical study. Spine (Phila Pa 1976). 2009;34:E9-15. doi: 10.1097/ BRS.0b013e318188386a.
25. Andreshak JL, Dekutoski MW. Management of unilateral facet dislocations: a review of the literature. Orthopedics. 1997;20:917-926.

26. Bucholz RD, Cheung KC. Halo vest versus spinal fusion for cervical injury: evidence from an outcome study. J Neurosurg. 1989;70:884-92.

27. Dvorak MF, Noonan VK, Fallah N, Fisher CG, Finkelstein J, Kwon $\mathrm{BK}$, et al. The influence of time from injury to surgery on motor recovery and length of hospital stay in acute traumatic spinal cord injury: an observational Canadian cohort study. I Neurotrauma. 2015;32:645-54.

28. Petitjean ME, Mousselard H, Pointillart V, Lassie $P$, Senegas J, Dabadie P. Thoracic spinal trauma and associated injuries: should early spinal decompression be considered? J Trauma. 1995;39:368-72.

29. Bartels RH, Donk R. Delayed management of traumatic bilateral cervical facet dislocation: surgical strategy: report of three cases. J Neurosurg. 2002; 97:362-5.

30. An HS. Cervical spine trauma. Spine (Phila Pa 1976). 1998;23:2713-29.

31. Brodke DS, Anderson PA, Newell DW, Grady MS, Chapman JR. Comparison of anterior and posterior approaches in cervical spinal cord injuries. J Spinal Disord Tech. 2003;16:229-35.

32. Kwon BK, Fisher CG, Boyd MC, Cobb J, Jebson H, Noonan V, et al. A prospective randomized controlled trial of anterior compared with posterior stabilization for unilateral facet injuries of the cervical spine. J Neurosurg Spine. 2007;7:1-12.

33. Cruz HY, Joaquim AF, Tedeschi H, Patel AA. Evaluation of the SLICS use in the treatment of subaxial cervical spine injuries. Arq Neuropsiquiatr. 2015;73:445-50.

34. Joaquim AF, Ghizoni E, Tedeschi H, Da Cruz HY, Patel AA. Clinical results of patients with subaxial cervical spine trauma treated according to the SLIC score. J Spinal Cord Med. 2014;37:420-4.

35. Fehlings MG, Vaccaro A, Wilson JR, Singh A, Cadotte WD, Harrop JS, et al. Early versus delayed decompression for traumatic cervical spinal cord injury: results of the Surgical Timing in Acute Spinal Cord Injury Study (STASCIS). PLoS One. 2012;7:e32037. doi: 10.1371/journal. pone.0032037.

36. Nagata K, Inokuchi K, Chikuda H, Ishii K, Kobayashi A, Kanai H, et al. Early versus delayed reduction of cervical spine dislocation with complete motor paralysis: a multicenter study. Eur Spine J. 2017;26:1272-6. 